\title{
Association between Non Dominant Hip and Dominant Shoulder Internal Rotation Range of Motion and Shoulder Injury in Cricket Fast Bowlers: A Cross-Sectional Study
}

\author{
Ajay Kumar ${ }^{1}$, Divyesh Arvindbhai Vaja ${ }^{2 *}$, Deepak Kumar Pradhan ${ }^{3}$
}

${ }^{1}$ Professor, Department of Physiotherapy, Srinivasa University, Mangalore, Karnataka, India

${ }^{2}$ Post-Graduate Student, Department of Physiotherapy, Srinivasa University, Mangalore, Karnataka, India

${ }^{3}$ Assistant Professor, Department of Physiotherapy, Srinivasa University, Mangalore, Karnataka, India

DOI: $10.36348 / J A S P E .2019 . v 02 i 07.001 \quad$ | Received: 28.08.2019| Accepted: 04.09.2019| Published: 29.09 .2019

*Corresponding author: Divyesh Arvindbhai Vaja

\section{Abstract}

Background: The overhead throwing motion is complex, and restrictions in range of motion (ROM) at the hip may place additional demands on the shoulder that lead to injury. However, the relationship between hip and shoulder ROM in fast bowlers with and without a history of shoulder injury is unknown. Aim: To find out the association between non dominant hip and dominant shoulder internal rotation range of motion with and without shoulder injury in cricket fast bowlers. Study design: A cross-sectional study. Method: 35symptomatic and 35 asymptomatic fast bowlers were included. Symptomatic and asymptomatic fast bowlers were screened for the measurement of non-dominant hip and dominant shoulder rotation range of motion. And SPADI used to screen for the shoulder injury in symptomatic fast bowlers. Results: There is significant association between shoulder injury and non-dominant hip IR ROM and no significant association between non-dominant hip and dominant shoulder IR ROM. Conclusion: Fast bowlers with shoulder injury had decreased non dominant hip IR ROM and the co-relation was significant. The decreased IR ROM was compensated by other kinematic chain and ER ROM. Hence, fast bowlers with shoulder pathology must always be screened for hip joint ROM as well.

Keywords: Fast bowlers, shoulder injuries, shoulder and hip range of motion.

Copyright @ 2019: This is an open-access article distributed under the terms of the Creative Commons Attribution license which permits unrestricted use, distribution, and reproduction in any medium for non-commercial use (NonCommercial, or CC-BY-NC) provided the original author and source are credited.

\section{INTRODUCTION}

Cricket is by far one of the most popular games and it would not be appropriate to consider these sports anything less than a religion. There has been a huge fan following but unfortunately the bowlers sustain a lot of injuries.

The myofascial connection of the shoulder complex and the trunk describe by, Latissimus dorsi to serratus anterior and ipsilateral rhomboid and external oblique muscle, and contralateral internal oblique, and femoral adductor muscle. The serratus anterior muscle courses anteriorly around the rib cage to attach to the ribs and interdigitates with the external oblique. Then it continues with the internal oblique and femoral adductor muscle on the contralateral side. The orientation of these muscles, anatomically linking the upper extremity, trunk, and the lower extremity across the front of the body and back of the body linkage by latismus dorsi and contralateral gluteus maximus via thoracolumbar fascia [1].

According to studies, it's proven that any restriction in internal rotation (IR) of the non-dominant hip during the follow-through phase of the throwing motion may limit the lower extremity's ability to absorb or dissipate the energy generated during the acceleration phase. In turn, this may place greater demands on the rotator cuff to act as a brake to decelerate the arm during follow-through and thus lead to posterior shoulder dysfunction and rotator cuff injury [2].

Non-dominant hip internal rotation restriction puts more demands on the dominant arm during follow through phase of fast bowling in fast bowlers which may limits the lower extremity's ability to absorb and dissipate the energy from lower extremity to upper extremity. In turn, this may place greater demands on the rotator cuff to act as a brake to decelerate the arm 
during follow-through and thus lead to posterior shoulder dysfunction and rotator cuff injury [2]. But no studies have been attempted to find out the relationship between these two factors in cricket fast bowlers, so the present study aimed to find out the relationship exists between non dominant hip internal rotation and dominant shoulder internal rotation range of motion and shoulder injury among cricket fast bowlers with history of shoulder injury and with no history of shoulder injury.

\section{AIMS OF THE STUDY}

To find out the association between non dominant hip and dominant shoulder internal rotation range of motion with and without shoulder injury in cricket fast bowlers.

\section{METHODOLOGY}

STUDY DESIGN: A cross sectional study

STUDY POPULATION: Fast bowlers between 13-28 years.

SAMPLING METHOD: Purposive sampling.

SAMPLE SIZE: 70

STUDY SETTING: Cricket clubs in Gujarat and Mangalore.

STUDY DURATION: From April 2018 to May 2019

\section{CRITERIA FOR SELECTION INCLUSION CRITERIA}

Inclusion criteria [3-6]: (for Group A-Symptomatic)

- Fast bowlers (defined by the coach).

- Fast bowlers with 1-year of experience.

- Fast bowlers who are currently suffering of shoulder injury.

- Age: 13 to 28 years.

- Negative Craig's test.

Inclusion criteria [3-6]: (for Group B Asymptomatic)

- $\quad$ Fast bowlers (defined by the coach).

- Fast bowlers with 1-year of experience.

- Age: 13 to 28 years.

- Negative Craig's test.

- Fast bowlers with no current musculoskeletal pain and injury.

\section{Exclusion criteria [3, 7]:}

- Use of steroids and other performanceenhancing drugs.

- Diabetes.

- Previously upper limb fracture or any surgeries within past 1 year.

- Neurological disorder like cervical and lumbar radiculopathy.
- Those who regularly participate in overhead sport other than cricket.

\section{TESTER}

Principal investigator was a qualified physiotherapist and currently perusing master of physiotherapy degree in musculoskeletal and sports conditions in Srinivas College of physiotherapy \& research Centre, Mangalore.

\section{TESTING EQUIPMENT}

- Smartphone clinometer

- SPADI scale

\section{PROCEDURE}

\section{Method of Collection of Data}

Cricket fast bowlers were screened from various sports clubs in and around Mangalore and Gujarat. Followed by this, bowlers were included in the study based on the selection criteria. The participants were then asked to sign informed consent prior to voluntary participation in the study.

- Group A (Symptomatic subjects): Players with shoulder injury underwent the measurement of non-dominant hip and dominant shoulder range of motion.

- Group B (Asymptomatic subjects): Players without shoulder injury underwent the measurement of non-dominant hip and dominant shoulder range of motion.

\section{Measurement procedure: (for Group A and Group B)}

Shoulder range of motion by smartphone clinometer application [8]

The bowlers were asked to lie in supine lying position and the shoulder joint was placed at ninety degrees of Shoulder abduction and elbow ninety degrees of flexion. A towel roll was placed under the proximal humerus to maintain alignment of the humerus to the midline of the subject's torso during testing. Inclinometer was placed onto the radial aspect of the forearm, just distal to the radial styloid process. For internal rotation players actively rotated the arm medially and for external rotation, players actively rotated the arm laterally. Stabilization of the scapula was applied by the therapist for both internal rotation and external rotation.

Hip range of motion by smart phone clinometer application [9, 10]

Players were positioned prone lying and the hip was kept in neutral in regards to abduction/adduction of the thigh and the pelvis stabilized to the table with a belt. The participant's arms were positioned at his/her side with the head in the position of most comfort. Inclinometer was placed at the midline of medial shaft of tibia between the medial malleolus and medial tibial condyle. For internal 
rotation, the knee of the leg were moved into $90^{\circ}$ flexion or perpendicular to the table and players move the shank of the leg out. For External rotation, players flexed one knee to 90 degrees and then the shank was moved towards the midline. The non-measure leg in extension and slightly abducted to allow for full measurement. The player's pelvis was stabilized by belt during rotation to prevent pelvic rotation.

\section{OUTCOME MEASURES}

- SPADI (Shoulder Pain and Disability Index)

- Smartphone Inclinometer

OBSERVATION AND RESULTS

Table-1: (Descriptive Analysis of Demographic Data)

\begin{tabular}{|l|l|l|}
\hline VARIABLES & Symptomatic & Asymptomatic \\
\hline Subjects(N) & 35 & 35 \\
\hline Age (Years) & $18.49( \pm 2.79)$ & $18.26( \pm 3.72)$ \\
\hline Height(cm) & $158.94( \pm 3.93)$ & $160.86( \pm 6.09)$ \\
\hline Weight(kg) & $60.60( \pm 4.35)$ & $59.51( \pm 4.71)$ \\
\hline BMI(kg/m $\left.{ }^{2}\right)$ & $23.98( \pm 1.29)$ & $23.01( \pm 1.44)$ \\
\hline Experience of year & $3.51( \pm 1.73)$ & $2.69( \pm 1.45)$ \\
\hline Dominance & $25 / 10$ & $28 / 7$ \\
& R/L & $\mathrm{R} / \mathrm{L}$ \\
\hline BMI= Body Mass Index, R=Right, L=Left & \\
\hline
\end{tabular}

Table-2: Pearson Correlation between SPADI, SIR, HIR AND SER

\begin{tabular}{|l|l|l|l|}
\hline Players & VARIABLE & PEARSON (r) \\
\hline With shoulder injury & SPADI with HIR & -.568 & P VALUE \\
\cline { 2 - 4 } & SPADI with HER & -.085 & 0.000 \\
\cline { 2 - 4 } & SPADI with SIR & -.434 & 0.627 \\
\cline { 2 - 4 } & SPADI with SER & -.134 & 0.009 \\
\cline { 2 - 4 } & HIR with SIR & 0.193 & 0.266 \\
\cline { 2 - 3 } & HIR with SER & -0.083 & 0.635 \\
\cline { 2 - 3 } & HER with SIR & 0.226 & 0.191 \\
\cline { 2 - 3 } & HER with SER & 0.350 & 0.294 \\
\hline \multirow{5}{*}{ Without shoulder injury } & HIR with SIR & 0.258 \\
\cline { 2 - 3 } & HIR with SER & -.047 \\
\cline { 2 - 3 } & HER with SIR & -.010 \\
\cline { 2 - 3 } & HER with SER & 0.135 \\
\hline
\end{tabular}

SPADI- Shoulder Pain And Disability Index, SIR= Shoulder Internal Rotation, SER= Shoulder External Rotation, HIR= Hip Internal Rotation, HER=Hip External Rotation

Graph-1: Demographic Data

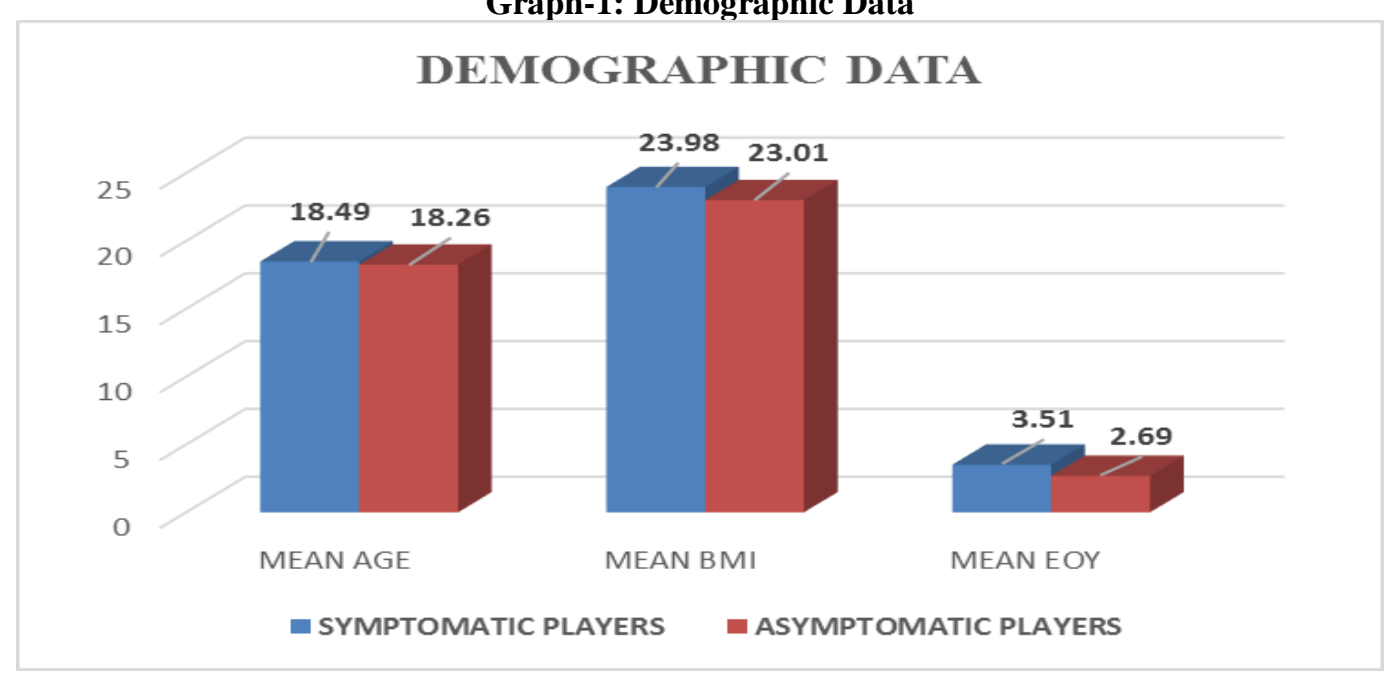




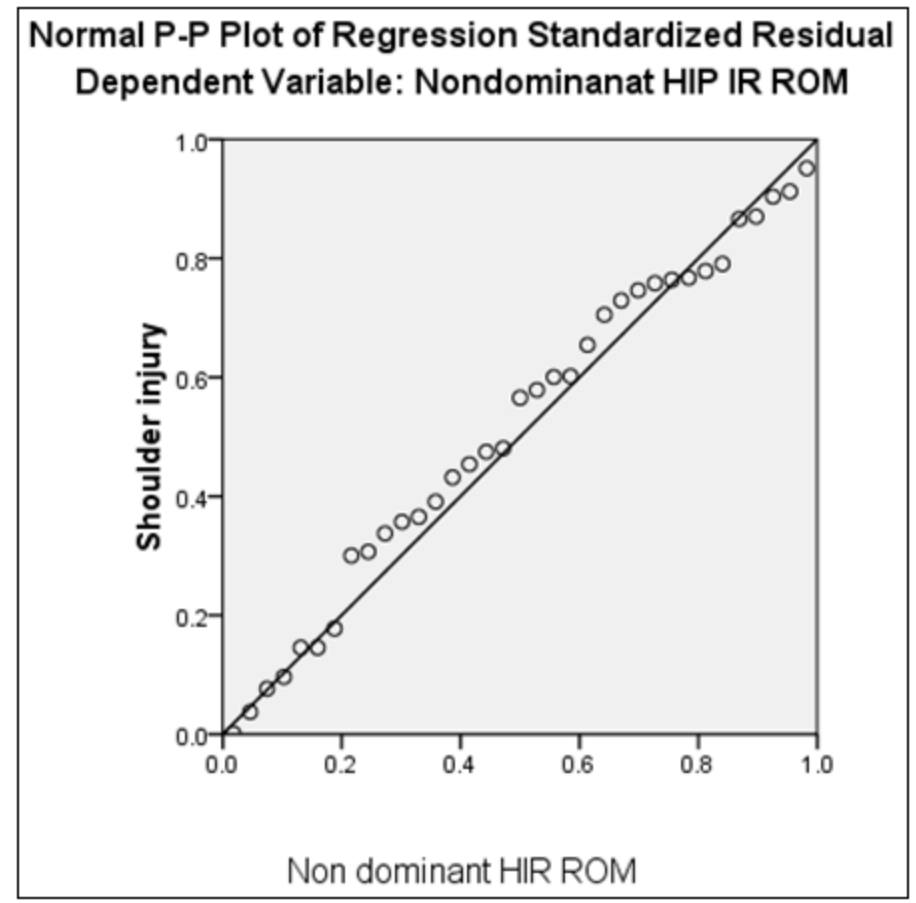

Graph-2: Regression Analysis

\section{DISCUSSION}

The aim of our study was to find out the association between non dominant hip and dominant shoulder internal rotation range of motion and shoulder injury in fast bowlers. 70 fast bowlers with or without shoulder pain age between13-28 were included in this study.

Present study focused on influence of nondominant hip internal rotation restriction on shoulder injury and dominant shoulder internal rotation ROM. During throwing motions, energy required during ball release is transferred through the body especially from the lower limb and trunk. Researchers found that there is an association between the lower limb biomechanics and ball release in fast bowlers. So any restriction or difficulty during ball release, particularly in followthrough phase is not only because of shoulder pathology it could be because of altered lower limb kinematics as well [11].

Scher et al., [2] concluded that IR of the nondominant hip may be responsible for decelerating the athlete's body. Thus, it is plausible that a lack of IR in the non-dominant hip may transfer some of the demands of decelerating the body from the hip to the shoulder, thereby dissipating less force through the trunk and increasing forces at the shoulder. In turn, the athlete with limited non-dominant hip IR may be predisposed to shoulder injury.

Kibler et al., [11] concluded that any hip rotational abnormalities need to be evaluated. Because any inability to achieve normal motions or generate normal velocity in the lower extremity affect the transfer of force to the scapula and the arm and can lead to shoulder injury.

All previous studies has identified the relationship between shoulder, trunk and lower limb through kinetic chain passing posteriorly and obliquely from lower limb to opposite shoulder. During throwing motion, hip rotational range of motion deficit leads to shoulder injury and alter shoulder rotation motion in fast bowlers. So this study aimed to find out the association between non dominant hip and dominant shoulder IR range of motion and shoulder injury in cricket fast bowler.

Considering the scarcity of evidence on hip rotation motion and shoulder injury in fast bowlers, we attempted to investigate association between non dominant hip and dominant shoulder internal rotation ROM and shoulder injury among cricket fast bowlers. Present study revealed that there was moderate negative co-relation $(\mathrm{r}=0.568, \mathrm{p}=0.00)$ between shoulder injury and non-dominant hip internal rotation ROM and there was no significant correlation $(\mathrm{r}=0.193)$ between shoulder and hip rotation ROM in both injured and noninjured cricket fast bowler. So, it can be hypothesized that altered lower limb and trunk kinematics can predispose to shoulder pathology. The kinematic chain connecting the lower extremity to the opposite shoulder may be the possible cause. Kinematic chain connects the lower extremity, trunk, shoulder and scapula through muscles and fascia (lattisimus dorsi to contralateral gluteus maximus via thoraco lumbar fascia) [11] and this connection between the shoulder and scapula is the pivoting link [1]. 
In this study, we could not find significant correlation between shoulder and hip rotation ROM in both symptomatic and asymptomatic group which could be because of compensation in total arc of shoulder and hip ROM at the joint or compensation at trunk or scapular positions during the functional activity. So, it can be hypothesized that non-dominant hip IR cannot alter the dominant shoulder IR alone and there could be some other confounding factors like dominant hip range of motion, scapular alterations, trunk rotation motion and altered biomechanics of hip and trunk [12].

The cross-sectional design does not provide the cause-and-effect relationship. Only male bowlers were included and assessor blinding was not done in this study. Hip and shoulder ROM were measured under static conditions and not during the throwing motion. So, further investigations on the same are suggested by measuring ROM of shoulder and hip during dynamic phase rather than static and also high level study can be done on effectiveness of hip joint mobilization on shoulder outcomes.

\section{CONCLUSION}

This study concluded that fast bowlers with shoulder injury had decreased non dominant hip IR ROM and the co-relation was significant. Though there was decrease IR ROM of non-dominant hip and dominant shoulder, it was compensated by other kinematic chain and ER ROM. Hence, fast bowlers with shoulder pathology must always be screened for hip joint ROM as well.

\section{REFERENCES}

1. Kaur, N., Bhanot, K., Brody, L. T., Bridges, J., Berry, D. C., \& Ode, J. J. (2014). Effects of lower extremity and trunk muscles recruitment on serratus anterior muscle activation in healthy male adults. International journal of sports physical therapy, 9(7), 924-937.

2. Scher S, Anderson K, Weber N, Bajorek J, Rand K, Bey MJ. Associations among hip and shoulder range of motion and shoulder injury in professional baseball players. Journal of athletic training. 2010 Mar;45(2):191-197.
3. Kumar, S., Kulandaivelan, S., Kaur, J., Chaturvedi, R., Girdhar, B., Singh, V., ... \& Kumar, V. (2015). One year prevalence of musculoskeletal disorder among cricket Players in Haryana: A retrospective study. International Journal Phy Education Sports health, 2015; 2(2):75-77.

4. Ranson, C., \& Gregory, P. L. (2008). Shoulder injury in professional cricketers. Physical Therapy in Sport, 9(1), 34-39.

5. Ranson, C. A., Burnett, A. F., King, M., Patel, N., \& O'Sullivan, P. B. (2008). The relationship between bowling action classification and threedimensional lower trunk motion in fast bowlers in cricket. Journal of sports sciences, 26(3), 267-276.

6. Magee, D. J. (2008). Orthopedic physical assessment. 5th ed; Edmonton (Canada) Reed Elsevier; 681-83.

7. Sundaram, B., Bhargava, S. K. N., \& Karuppannan, S. (2012). Glenohumeral rotational range of motion differences between fast bowlers and spin bowlers in elite cricketers. International journal of sports physical therapy, 7(6), 576-585.

8. Smith, A. B. (2016). Validation Of A Smartphone Application For Measuring Shoulder Internal Rotation And External Rotation Range Of Motion With Intra-Rater Reliability. Available from: http://digitalcommons.otterbein.edu/stu_master

9. Bremner, C. B. (2013). The Acute Effect of Two Hip External Rotators Stretchers on Range of Motion. Univ Nevada. Available from https://digitalscholarship.unlv.edu/thesesdissertatio ns

10. Charlton, P. C., Mentiplay, B. F., Pua, Y. H., \& Clark, R. A. (2015). Reliability and concurrent validity of a Smartphone, bubble inclinometer and motion analysis system for measurement of hip joint range of motion. Journal of Science and Medicine in Sport, 18(3), 262-267.

11. Ben Kibler, W. (1998). The role of the scapula in athletic shoulder function. The American journal of sports medicine, 26(2), 325-337.

12. Wilk, K. E., Obma, P., Simpson, C. D., Cain, E. L., Dugas, J., \& Andrews, J. R. (2009). Shoulder injuries in the overhead athlete. Journal of orthopaedic \& sports physical therapy, 39(2), 3854. 